\title{
Toward an Intelligent Driving Behavior Adjustment Based on Legal Personalized Policies Within the Context of Connected Vehicles
}

OPEN ACCESS

Edited by:

Sakdirat Kaewunruen,

University of Birmingham,

United Kingdom

Reviewed by:

Maria Vittoria Corazza,

Sapienza University of Rome, Italy

Samuel Labi,

Purdue University, United States

*Correspondence:

Nafaa Jabeur

nafaa.jabeur@gutech.edu.om

Specialty section:

This article was submitted to

Transportation and Transit Systems,

a section of the journal

Frontiers in Built Environment

Received: 27 March 2021

Accepted: 31 May 2021

Published: 17 June 2021

Citation:

Outay F, Jabeur N, Haddad H, Bouyahia $Z$ and Gharrad H (2021) Toward an Intelligent Driving Behavior

Adjustment Based on Legal Personalized Policies Within the Context of Connected Vehicles. Front. Built Environ. 7:686732. doi: 10.3389/fbuil.2021.686732

\author{
Fatma Outay $^{1}$, Nafaa Jabeur ${ }^{2 *}$, Hedi Haddad ${ }^{3}$, Zied Bouyahia ${ }^{3}$ and Hana Gharrad ${ }^{2,4}$ \\ ${ }^{1}$ College of Technological Innovation, Zayed University, Dubai, United Arab Emirates, ${ }^{2}$ Department of Computer Science, German \\ University of Technology in Oman (GUtech), Muscat, Sultanate of Oman, ${ }^{3}$ Department of Computer Science, Dhofar University, \\ Salalah, Sultanate of Oman, ${ }^{4}$ Transportation Research Institute (IMOB)-Hasselt University, Hasselt, Belgium
}

The advent of Connected Vehicles (CVs) is creating new opportunities within the transportation sector. It is, indeed, expected to improve road traffic safety, enhance mobility, reduce fuel consumption and gas emissions, as well as foster economic growth via investments and jobs. However, to motivate the deployment of $\mathrm{CVs}$ and maximize their related benefits, policymakers must create appropriate neutral legal frameworks. These frameworks should promote the innovation of current road infrastructures, support cooperation and interoperability between transportation systems, and encourage fair competition between companies while upholding consumer privacy as well as data protection. We argue that policymakers should also support innovative mobility services toward a better accommodation of individual drivers and vehicles. Within this scope, we are proposing in this paper an intelligent approach that promotes the implementation of personalized road policies based on driving behaviors, driving performance, and the ongoing road traffic situation. These policies, which are dynamic in space and time, ultimately aim to increase drivers' awareness by encouraging behavioral self-regulation. To meet our goals, we are using software agents that autonomously manage the driving behaviors according to well-defined transitions between driving states while enabling appropriate message exchanges between CVs. We run software simulations as well as field tests and obtained promising results that would reflect the relevance of implementing our vision of personalized policies.

Keywords: intelligent transportation system, connected vehicles, personalized policies, driving behaviors, beliefdesire-intension, behavioral self-regulation

\section{INTRODUCTION}

The concept of Intelligent Transportation System (ITS) is conventionally about technologies that endow the components of a national transportation system infrastructure (e.g., traffic lights, bridges, etc.) with intelligent mechanisms via embedded sensors and enhanced communication capabilities (Ezell and Atkinson, 2015). These capabilities have particularly led to V2X (i.e., Vehicle-2-Vehicle, Vehicle-2-Infrastructure, etc.) solutions that are remarkably capable of enhancing operational performance, safety, and environmental benefits (Ezell and Atkinson, 2015). They have also led to the emergence of the promising concept of Connected Vehicles (CVs), which will most probably 
become a fundamental component of the traffic stream in the upcoming few years (Pariota et al., 2017). More specifically, CVs refers to the technologies, services, and applications that connect a given vehicle to its surroundings, including external devices, neighboring vehicles, road infrastructure elements, and networks. Its introduction has resulted in a significant positive impact on capacity and traffic operations, travel demands and habits, and mobility behaviors (Pariota et al., 2017). Indeed, as reported in (Jadaan et al., 2017), CV applications can: 1) address $81 \%$ of unharmed driver crashes in highway, particularly since vehicles can sense and communicate the hazards around them; 2 ) enable users and system operators to make on-the-fly smarter choices to reduce delay during traffic jams; and 3) contribute to green transportation choices that reduce vehicle emissions in pollutants, particularly through their real-time advisories on fuel consumption and current road traffic. In spite of these advantages, the investigation of this impact particularly on human driving behaviors remains of paramount importance (Jadaan et al., 2017). This statement is support by the European report on accident research and safety (Annual Accident Report, 2017)[4] showing that about 30\% of emerging road accidents are due to environmental factors (e.g., poor visibility, weather conditions, slippery roads, etc.), $10 \%$ are related to vehicles (e.g., bad maintenance, flat tires, etc.), and $90-95 \%$ are due to human factors.

Driving behaviors have been long studied in transportation research (e.g., Zhu et al., 2013; Jabbar et al., 2020; K. W. Lee et al., 2018; Alkinani et al., 2020; Tanveer et al., 2020), to understand, identify, detect, and predict drivers' actions that cause and/or would cause road traffic crashes. In order to identify the set of factors that would impact driving performance with respect to given situations, driving behaviors have been classified into operational, tactical, and strategic (Abuali and Abou-Zeid 2016). For each class of behavior, several models have been proposed (Toledo 2007). Within the context of CVs, several research works (e.g., Tampère et al., 2009; Kesting et al., 2010,[14]) have proposed models that attempted to realistically include drivers' behaviors and performance in the evaluation of related applications, particularly since drivers cannot simply follow these applications blindly. In spite of the proven results, additional works are still needed to thoroughly investigate driving behaviors and ultimately assess the readiness of drivers, road infrastructures, vehicles, and policies (Tampère et al., 2009). In this regard and due to the absence of proactive public policies, the development and adoption of $\mathrm{CV}$ technologies continue to be slow and the full benefits of CVs will not be attained (Talebpour and Mahmassani 2016). Appropriate policies must, therefore, be implemented to mainly regulate the use of $\mathrm{V} 2 \mathrm{X}$ infrastructure, protect the privacy and security of drivers' data, ensure regulations are technology neutral, and allow vehicle owners to access and use their own data (Mcquinn and Castro 2018). These policies are of paramount importance as CVs are expected to have a significant impact on current planning and land-use models (Ilgin Guler et al., 2014), on geometric design of highways, as well as on roads and signalized intersections (Jadaan et al., 2017).

Furthermore, several research works (e.g., Mcquinn and Castro 2018; Choi et al., 2016) have highlighted the importance of policies/initiatives related to drivers' selfregulation as they increase public trust, decrease risks related to the adoption of new technologies, and enable the creation of a more flexible regulatory environment, especially where existent standards are rigid (Gao and Chen 2019). As such, we argue in this paper that self-regulations would be an important booster of $\mathrm{CV}$ services and technologies based on driving behaviors and performance. More specifically, we are aiming to address the following question: Can we create a new framework within which drivers are allowed to behave as per a set of dynamically changing policies that fit their individual driving behavior/performance, vehicle characteristics, current road infrastructure, and ongoing contextual events? To address this gap in the literature, we are proposing in this paper an intelligent approach that enables the implementation of legal personalized policies and promotes selfregulation behaviors. Legal personalized policies are defined as specific road traffic rules tailored to individual driving behaviors. In order to improve road traffic safety and enhance mobility, our approach is particularly enabling the exchange of relevant variable messages among CVs depending on the ongoing situation (e.g., slippery road due to heavy rain or slow traffic flow), driver's behaviors (e.g., unsafe right turns, harsh braking), road infrastructure (e.g., speed changed due to road works), etc. The ultimate goal of our work is to enable an intelligent driving behavior adjustment based on personalized policies. More precisely, we are aiming to provide drivers with feedbacks generated by intelligent software entities to enable them modify their driving behaviors toward safer commutes. The contributions of our approach could be summarized as follows: 1) A new framework that focuses on implementing personalized road traffic policies (based on driving parameters, including driving performance, road infrastructure, etc.); and 2) A new well-defined driving behavior model within which transitions between driving states are intelligently determined and relevant data are shared among CVs accordingly.

In the reminder of the paper, Related Work outlines the current literature on driving behavior identification, assessment, and classification with a focus on the specific context of CVs. It also highlights the need to address the untapped issue of policies that must be implemented to support the expected growing field of CVs. Intelligent Adjustment of Driving Behavior Based on Personalized Policies is dedicated to our proposed solution where we explain the fundamentals of our vision as well as the agent-based approach for the management of driving behaviors. Research Design and Results sheds light on the setup and the results of our simulations and field tests.

\section{RELATED WORK}

Individual driving behaviors, including acceleration, deceleration, and aggressive driving have been intensively studied in transportation safety research (Zhu et al., 2013). Based on their timescales, these behaviors can be classified as operational (include actions executed over less than a second, basically to stay safe or respect traffic regulations), tactical 
(include a set of operational maneuvers to meet a short-term goal, like turns, stops, and lane changes), and strategic (define the actions to reach long-term goals, like route calculation and selection of transportation modes) (Abuali and Abou-Zeid 2016). Efforts have focused on understanding, identifying, detecting, and predicting the driving styles, mainly by investigating human factors (Chan et al., 2020). These factors have been categorized into six classes (i.e., physiological factors, driving skills, driving desires, personality traits, imperfect driving, and socio-economic factors (Sharma et al., 2018). They have particularly been used to detect potentially dangerous driving situations, based on image and video processing techniques whereby drivers' faces are captured and analyzed (Alkinani et al., 2020). In this regard, the authors in (Jabbar et al., 2020) have proposed an approach based on Convolutional Neural Networks (CNN) techniques to detect and classify driver drowsiness. The drowsiness classification is performed using facial landmarks detected by a camera. In (K. W. Lee et al., 2018), the authors have proposed an approach for the detection of aggressive driving emotions based on CNN. The approach uses NIR cameras for the discovery of facial feature points and the measurement of their changes. It also uses thermal cameras to measure the changes of driver's temperature. In (Tanveer et al., 2020), the authors have attempted to build a model to express drivers' acceleration behaviors under panic. To this end, they have proposed an enhanced Intelligent Driver Model (IDM) to observe the continuous changes in acceleration and deceleration.

To increase safety and encourage safer driving behaviors, driving aids have been proposed in several research works. Indeed, in (Ali et al., 2020), the authors have found that this approach of driver notification trough the connected environment improve the behavior of the driver in terms of safety when communications are well-established. In (Chang et al., 2019), the effect of an onboard unit (OBU) as a V2V technology has been analyzed in different traffic scenarios. Based on the analysis presented in their work, the authors found that the use of onboard units has a positive impact on drivers' driving behaviors. In (Vaezipour et al., 2017), the authors have carried out an explorative qualitative study to identify the potential benefits of using a driver-centered solution where safe and ecological feedbacks and advices are provided to drivers about their driving behaviors via dedicated Human Machine Interfaces (HMIs). The authors have reported on their findings that their approach considerably improve road traffic safety. They have also highlighted the importance of designing dedicated HMIs to minimize drivers' distractions on roads. These results are supported by other studies, including (Birrell et al., 2014; Sanguinetti et al., 2020). Indeed, in (Birrell et al., 2014), the authors have presented a smart driving aid system and described its related results obtained from field tests based on an instrumented vehicle in a mixed-route. These results show that the proposed system can significantly improve road traffic safety as well as fuel efficiency by limiting the use of lower gears.

Within the field of CVs, several solutions have (implicitly or explicitly) addressed human driving factors. These solutions can be categorized based on how they modify drivers' behaviors (Talebpour and Mahmassani 2016), or more accurately how they influence driving behaviors toward safer commutes (Jadaan et al., 2017) (Table 1). In this regard, the authors in (Park et al., 2019) have considered the situation of reduced visibility and assessed the effectiveness of in-vehicle warning and assistance information design. The authors have used the Kansei engineering methods (Nagamachi 2003) to understand the emotions of drivers while under stress and identify the characteristics of the warnings that should be adapted accordingly. In (Qi et al., 2020), the authors have evaluated the performance of a Variable Speed Limit (VSL) system using a hierarchical framework under a CV environment. To this end, they have investigated the safety and the operational impact of the VSL system on the driver maneuvering behavior and then have performed statistical tests accordingly. In (Goli et al., 2018), the authors have used the data collected from V2V and V2I communications concerning road and traffic data to address the issue of predicting long-term locations for collision avoidance. A Gaussian Process Regression (GPR) approach is then used to identify drivers' motion patterns. In (Gong and Du 2018), the authors have proposed a merging strategy based on cooperative driving behaviors. The approach aims to create virtual vehicle platoons in order to ensure the safety and mobility of vehicles as well as the smoothness and stability of traffic flow. In (Dong et al., 2019), the authors have proposed a hierarchical velocity profile optimization strategy to influence driving behavior for the ultimate goal of reducing fuel consumption with the presence of several traffic lights. In (Yu et al., 2020), the authors have presented a mobility-aware approach based on a proactive edge-caching scheme with federated learning to improve cache performance and protect vehicles' privacy. In (D'Angelo et al., 2020), the authors have described a multidimensional approach for the detection of attacks on CVs based on the messages exchanged between vehicles. The approach includes an algorithm that implements a data-driven anomaly detection system as well as an algorithm to carry out on-the-fly classification of messages (into licit or illicit) for early alerting in the presence of malicious usages.

In the existing $\mathrm{CV}$ solutions that have addressed human driving factors, several technical, ethical, and legal issues have been raised. Appropriate policies and regulations are, therefore, needed in order to support the expected growth of the CV field. This statement was supported by the authors in (Mcquinn and Castro 2018) who have listed eight principles that policymakers should consider to motivate the deployment of CVs and maximize their related benefits. These principles are summarized as follows:

(1) Support V2X infrastructure

(2) Promote cooperation and interoperability for V2X Systems

(3) Incentivize companies to protect consumers from harm by ensuring that companies focus adequately on safety, promote fair competition, and uphold consumer protections

(4) Ensure regulations are technology neutral (i.e., neither favor nor disadvantage any $\mathrm{CV}$ technology) to create a level playing field for innovation

(5) Rely on transparent industry-led standards for data protection (i.e., policymakers should focus on voluntary 
TABLE 1 | Classification of the impact of $\mathrm{CV}$ solutions on driving behaviors.

\section{Class}

Description

Classification based on how drivers' behaviors are modified (Talebpour and Mahmassani 2016)

Event-based driver behavior adjustment Continuous driver behavior adjustment

Semi-automated/Automated driving
One-time audio and/or visual feedback is conveyed to drivers as instructions concerning an upcoming event Drivers are provided with continuous instructions to adapt their behaviors for a specific goal like reduce fuel consumption or implement safer lane changing

Autonomous vehicles and CV are both combined to produce more efficient traffic systems

Classification based on how drivers' behaviors are influenced toward safer commutes (Jadaan et al., 2017)

Safety solutions

Mobility solutions

Environmental solutions
Increase situational awareness and reduce/eliminate crashes via driver advisories and warnings (Ali et al., 2020) Try to optimize the use of networking capabilities for better wireless traffic detection Intend to advise drivers about how to reduce fuel consumption and its impact based on driving styles and traffic conditions self-regulatory principles for the auto industry to protect the cybersecurity and privacy of vehicle owners (Castro 2011)

(6) Restrict the increasing interference of regulators who are supervising $\mathrm{CV}$ privacy on the pretext of digitization

(7) Permit after-market modifications and repairs while protecting copyright holders' rights

(8) Allow vehicle owners to access and use their own data, which include diagnostic reports, geolocation information, purchasing records, and entertainment preferences.

The abovementioned principles are supported by the report published by the Victoria Transport Policy Institute (D. Lee 2021). This report has particularly pointed out that it would be difficult to commercialize the purchase of fully autonomous cars before 2050 in the absence of appropriate road infrastructures and regulations. Indeed, roadway development and maintenance will possibly need to change significantly to ensure optimal autonomous vehicle performance (Baker and Wagner 2013). Additional CV policyrelated issues have been investigated in (Hussain and Zeadally 2019). The authors have mainly highlighted the importance to create legal frameworks that support innovative mobility services toward a better accommodation of individual drivers/vehicles. As vehicles do not have the same capabilities and drivers do not have the same attributes (i.e., physiological factors, driving skills, driving desires, personality traits, etc.), we propose in this paper to consider the implementation of intelligent solutions that personalize road traffic policies based on driving performance while promoting selfregulation driving behaviors.

\section{INTELLIGENT ADJUSTMENT OF DRIVING BEHAVIOR BASED ON PERSONALIZED POLICIES}

\section{Vision Fundamentals}

Based on our thorough literature review, we argue that CV-related impact on road traffic and infrastructure as well as on drivers' behaviors still need extensive investigations. We also argue that dedicated tools to share relevant data among CVs and ultimately implement self-regulation driving behaviors and personalized policies would boost innovation and create trust on the provided services. Personalized policies refer here to the specific policies that should be applied to a given driver based on his/her driving performance, contextual conditions, vehicle characteristics, and any requirements from policymakers (policymakers may include the company that owns the vehicle, if applicable). These policies should be supported by the Behavioral Self-Regulation (BSR) of the driver. BSR can be defined, here, as the ability of this driver to act in his/her long-term preeminent interest while being consistent with his/her genuine values (Doğan et al., 2011). It mainly includes four components (Vohs et al., 2005): 1) Standards of desirable behavior; 2) Motivation to meet standards; 3) Monitoring of situations (i.e., how the driver is dealing with driving situations) and thoughts that precede breaking standards (i.e., what are the options that the driver may think about in order to deal with a driving situation before braking); and 4) Willpower allowing one's internal strength to control urges.

In order to include the concepts of personalized policies and BSR in our solution, we depict in Figure $\mathbf{1}$ our vision where the regular (or national) road traffic policies are represented as the umbrella of any other policy. In this vision, there might be a need for personalized policies in the following cases where the driver's driving behavior and performance are: (case a) Transgressing the national policies; (case b) Transgressing the specific policies of a given company (e.g., if the vehicle is part of a company fleet); (case c) Transgressing the national policies as well as the specific policies of a given company; and (case d) Seen to be dangerous and must be prohibited to prevent potential accidents. Except in case (e), specific policies may be applied to the driver. If this is the case then the driver must demonstrate BSR actions. Based on the decision of the policymakers, regular policies may be restituted, accordingly.

\section{System Model}

In order to implement our approach for an intelligent adjustment of driving behavior based on personalized policies within the context of CVs, we propose to model the driving states with respect to policies as follows (Figure 2):

- Normal-In this state, driving is bound to the common road traffic policies (i.e., national regulatory framework).

- Restitution-Based on the driving actions, if any road traffic policy is violated then the driving will transit to a Restitution state. In this case, the violation is assessed and classified. A recidivism level is calculated in order to check when, where, and how many times the driver has violated the same policy. If the fines related to the policy infraction is paid then the 


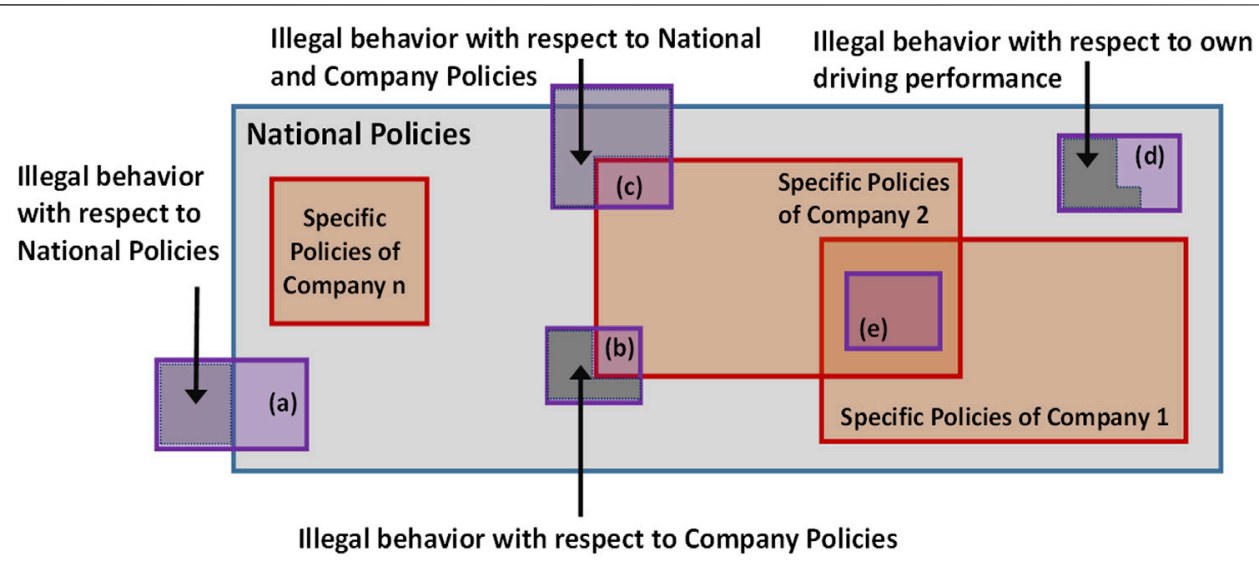

FIGURE 1 | Toward a framework of personalized road traffic policies.

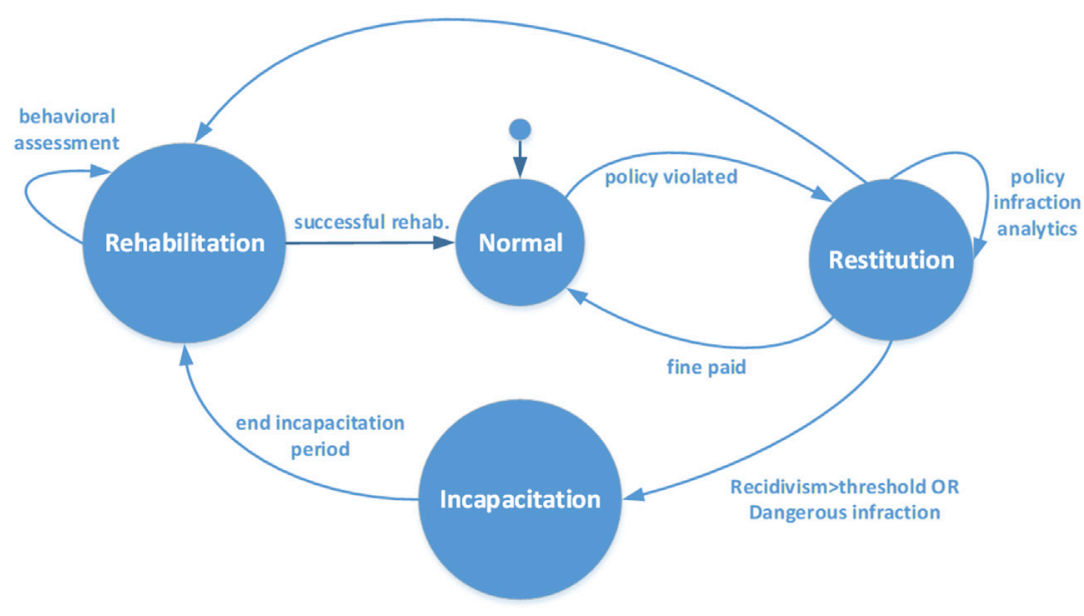

FIGURE 2 | Driving states with respect to road traffic policies.

driving state will return to Normal. If, as per the policymakers, the recidivism level exceeds a given threshold or if the policy infraction is classified to be highly dangerous then the state of the driving will become Incapacitation. The driving could also transit into a Rehabilitation state based on the analysis done during the Restitution state.

- Incapacitation-In this state, the driver is prevented from driving for a given period of time as per the rules specified by the policymakers.

- Rehabilitation-Once the incapacitation period is over, the driving will transit to a Rehabilitation state wherein personalized policies will be applied. During this state, the driving behavior (as well as the BSR) is assessed and a decision to return to the Normal state is made accordingly. The driving may also transit from Restitution to Rehabilitation as per the specific regulations of policymakers concerning policy infractions.
In order to meet our goals, we assume the followings:

- There are $n$ road traffic policies represented with $P=\left\{p_{1}, p_{2}, \ldots, p_{n}\right\}$

- There are $m$ road patterns $Q=\left\{q_{1}, q_{2}, \ldots, q_{m}\right\}$

- There are $k$ driving states represented with $S=$ $\left\{s_{1}, s_{2}, \ldots, s_{k}\right\}$ (in our case, $k=4$ as per Figure 2)

- A given commute $C$ is represented as a set of vectors $\left.C=\left\{<\mathrm{R}_{\mathrm{i}}, \mathrm{B}_{\mathrm{i}}, \pi_{\mathrm{i}}\right\rangle \mid \mathrm{i}=1, \ldots, \mathrm{m}\right\}$, where $R_{i}$ is a road section, $B_{i}$ is the vector (i.e., set) of driving behaviors in $R_{i}, \pi_{i}$ is the vector of policies applied to the road pattern of the road section $R_{i}$

For every road section, a behavioral assessment [i.e., $x_{i}=f\left(R_{i}, B_{i}, \pi_{i}, q_{i}\right)$ ] is performed in order to detect the compliance of the current driving behaviors (i.e., $B_{i}$ ) in the current road pattern (i.e., $q_{i}$ ) with the policies (i.e., $\pi_{i}$ ) applied to $q_{i}$. Based on this assessment, a new set of policies may be 


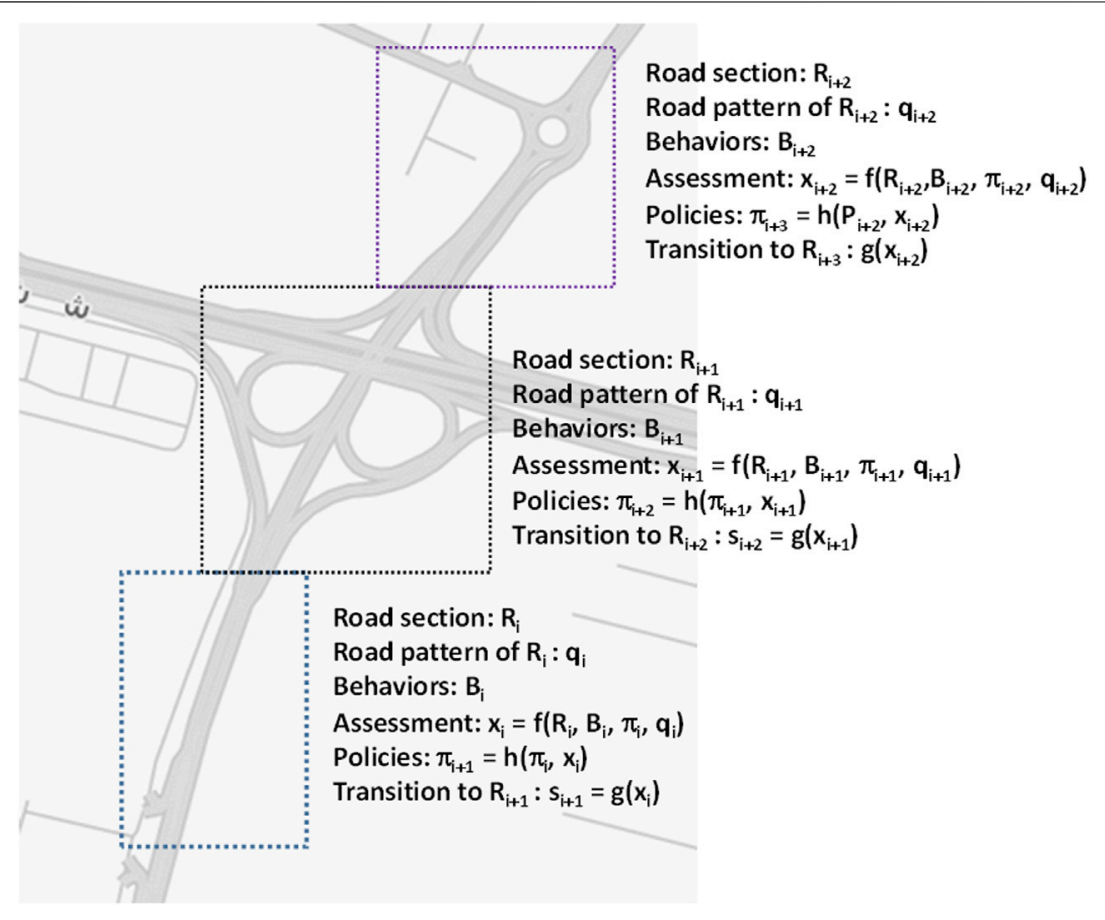

FIGURE 3 | Illustration of our system model concepts on a sample of road section.

generated [i.e., $\pi_{i+1}=h\left(\pi_{i}, x_{i}\right)$ ] as per the requirements of the policymakers. The assessment will also be used by a dedicated function [i.e., $s_{i+1}=g\left(x_{i}\right)$ ] in order to decide on the next driving state (see Figure 2) of the driving behavior. For the sake of illustration, we depict in Figure 3 the main concepts plotted on a selected road section.

\section{$A B \bar{D} I$ Architecture for the Management of Drivers' Behaviors}

The implementation of our approach will require from every vehicle to be intelligent and autonomous in order to assess the driving behaviors, examine the compliance of these behaviors with the current road traffic regulations, and infer the next driving state accordingly. To this end, we are proposing the use of software agents. Our choice is motivated by the success of these agents in intelligently and autonomously solve complex problems within highly dynamic, constrained, uncertain, and open environments (Djahel et al., 2020). More precisely, we are adopting a model inspired from the Belief-Desire-Intention (BDI) architecture which has been intensively used in the paradigm of Multi-Agent Systems (MAS). This architecture, which was successfully used to model humans, their reasoning, and their interactions (Norling 2004), includes three components: 1) Beliefs (refer to the information that the agent has about itself and the environment); 2) Desires (refer to the objectives or the situations that the agent would like to accomplish); and 3) Intentions (represent the actions that the agent has decided to perform). Our proposed architecture (Figure 4) is slightly different from the common BDI. We are, indeed, proposing a

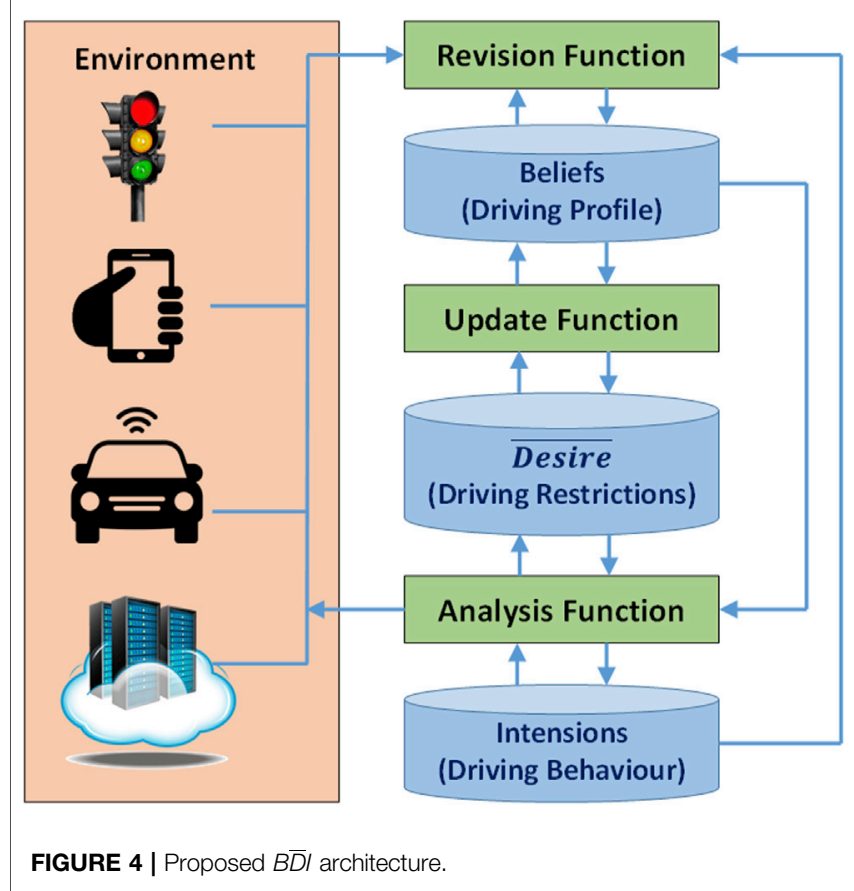

$B \bar{D} I$ architecture where the agent focuses on the actions that it must not accomplish and the situations that it must avoid. These actions are identified based on the regular and the personalized policies which are valid in every road section.

In order to explain the operation of our architecture, we assume a $B \bar{D} I$ agent is assigned to every vehicle. This agent 
can be located on the vehicle or on a remote server. Based on the data collected from the environment (i.e., from a dedicated mobile app, road infrastructure, CVs, or other online sources) as well as based on its current beliefs (e.g., current driving performance, driving policies in the current road section pattern, current driving state, etc.), the agent will run a Revision Function (RF) to update its knowledge. An Update Function (UF) is, then, executed to assess the driving performance based on the driving behavior, the current policies, and the current road pattern [i.e., $f\left(R_{i}, B_{i}, \pi_{i}, q_{i}\right)$ in Figure 3]. The UF function will also generate the new set of personalized policies [i.e., $h\left(\pi_{i}, x_{i}\right)$ in Figure 3].

In order to include self-regulation mechanisms and constrain drivers to improve their driving behaviors, we propose to calculate driving penalties for each driver. In this case, the agent will check the driving record of the driver as well as the current road traffic policies. The agent will then identify the regulations that have not been respected by the driver. It will also check the cases of recidivism (i.e., the cases where the driver violated the same policy several times). Penalties will then be calculated as follows:

$$
f_{u}(x)=f_{u-1}(x)+\sum_{i=1}^{n} c_{i} * r_{i} * \delta_{i}
$$

where $f_{u}(x)$ is the total penalty function until commute $u$. $f_{u-1}(x)$ is the total penalty until commute $u-1$. The parameter $n$ is the number of policies, $c_{i}$ denote the cost of violating the policy $i, r_{i}$ denote the recidivism factor for policy $i$, and $\delta_{i}=1$ if policy $i$ is violated during commute $u$ and $\delta_{i}=0$ otherwise.

Penalties can also be calculated per type of violation (e.g., overspeeding, usage of mobile phones, etc.). In this case, personalized trainings or warnings could be recommended or imposed to the driver, particularly when the driver is driving a company vehicle (the driving state will transit, in this case, to Rehabilitation as highlighted in Figure 2). Further investigations could be done in order to understand the reasons of road traffic violations in time and space. Within the specific context of CVs, the policymakers may consider to oblige the drivers whose penalties of violating specific rules exceed predefined thresholds to convey specific warnings to the follower CVs (e.g., drivers who are considered to have dangerous overpassing behaviors must notify other vehicles so they will be more cautious). Policymakers may also constraint these drivers to be in an Incapacitation state (see Figure 2). The investigation of these issues are out of the scope of this paper.

After executing the UF function (see Figure 4), the agent will execute an Analysis Function (AF) to identify the actions that the driver should do as self-regulation options, analyse his/her actual actions, and assess their impact on other CVs in the vicinity. The $\mathrm{AF}$ function is also responsible of determining the information that should be shared with the CVs and any other component of the ITS (e.g., road infrastructure). For example, for the specific case of Time To Collision (TTC), the commonly used equation (Eq. 2 below) could be updated with additional parameters (see Eq. 3) that reflect the skills of the driver in maneuvering his/her vehicle with respect to the speed and the current contextual information. In this case, the follower CVs could have a better estimation of the TTC and would exhibit well-informed reactions (e.g., be more cautious if the driver ahead is not able to control his/her vehicle appropriately at the current speed).

$$
T_{T C}=\frac{\left(X_{l}-X_{f}\right)-L_{l}}{\left(V_{f}-V_{l}\right)}
$$

Where $t$ is the time interval, $l$ is the leading vehicle, $f$ is the follower vehicle, $X$ is the position of the vehicle, $V$ is the speed, and $L$ is the length of the vehicle.

$$
T T C_{t}=\frac{\left(X_{l}-X_{f}\right)-L_{l}}{\left(V_{f} * p_{f}-V_{l} * p_{l}\right)}
$$

Where the parameter $p_{l}$ (alternatively $p_{f}$ ) represents the skills or the performance of the driver in controlling his/her vehicle at speed $V_{l}$ (alternatively $V_{f}$ ) under the contextual conditions at $t$.

As CVs may not have an extended context awareness, we propose to assign a software agent to every road section. This agent will collect information from all the vehicles crossing the section, the available proprietary or online traffic applications, as well as from sensors which are gathering environmental data. The agent will identify the actions that should not be carried out by the vehicles and broadcast, if necessary, updates on road traffic policies (e.g., during heavy rain, the maximum speed would be reduced from 80 to $60 \mathrm{~km} / \mathrm{h}$ ). The agent will also serve as support for the CVs during their commutes through the road section. This support as well as the security/privacy of related communications have been partially studied in our previous work (Mbarek et al., 2020). Additional details will appear in an upcoming publication.

\section{RESEARCH DESIGN AND RESULTS}

In order to test our solution, we run software simulations as well as field tests on a road section of Muscat, Oman (approximate coordinates: $\quad 23^{\circ} 33^{\prime} 52.8^{\prime \prime} \mathrm{N} \quad 58^{\circ} 25^{\prime} 09.0^{\prime \prime} \mathrm{E}, \quad 23^{\circ} 33^{\prime} 42.0^{\prime \prime} \mathrm{N}$ $58^{\circ} 25^{\prime} 36.3^{\prime \prime} \mathrm{E}$ ) (Figure 5A). Following the recommendations presented in (Feraud and Galland 2017), we selected the GAMA platform to run our simulations. On Figures 5B-D, the circular buffer around every vehicle depends on its current total penalties as well as on its current state (i.e., green, yellow, red, and blue for Normal, Restitution, Rehabilitation, and Incapacitation, respectively). This buffer visually reflects the obligation of the concerned vehicle to share information about its driving behavior/performance with the follower vehicles. For example, the vehicle $\mathrm{A}$ has transited from a Normal state (Figure 5B) to Restitution state (Figure 5C). The vehicle $G$ has transited from Restitution state (Figure 5B) to Rehabilitation state (Figure 5C). Furthermore, because the vehicle $\mathrm{C}$ exhibited dangerous/illegal behaviors on Figures 5B,C, its state transited from Rehabilitation to candidate to Incapacitation (Figure 5D). In our simulations, all the vehicles in Rehabilitation states send notifications to the neighboring CVs which are within a predefined notification range. This range is inversely proportional to the driver driving performance (i.e., the more the driver is behaving illegally and/or making infractions, 

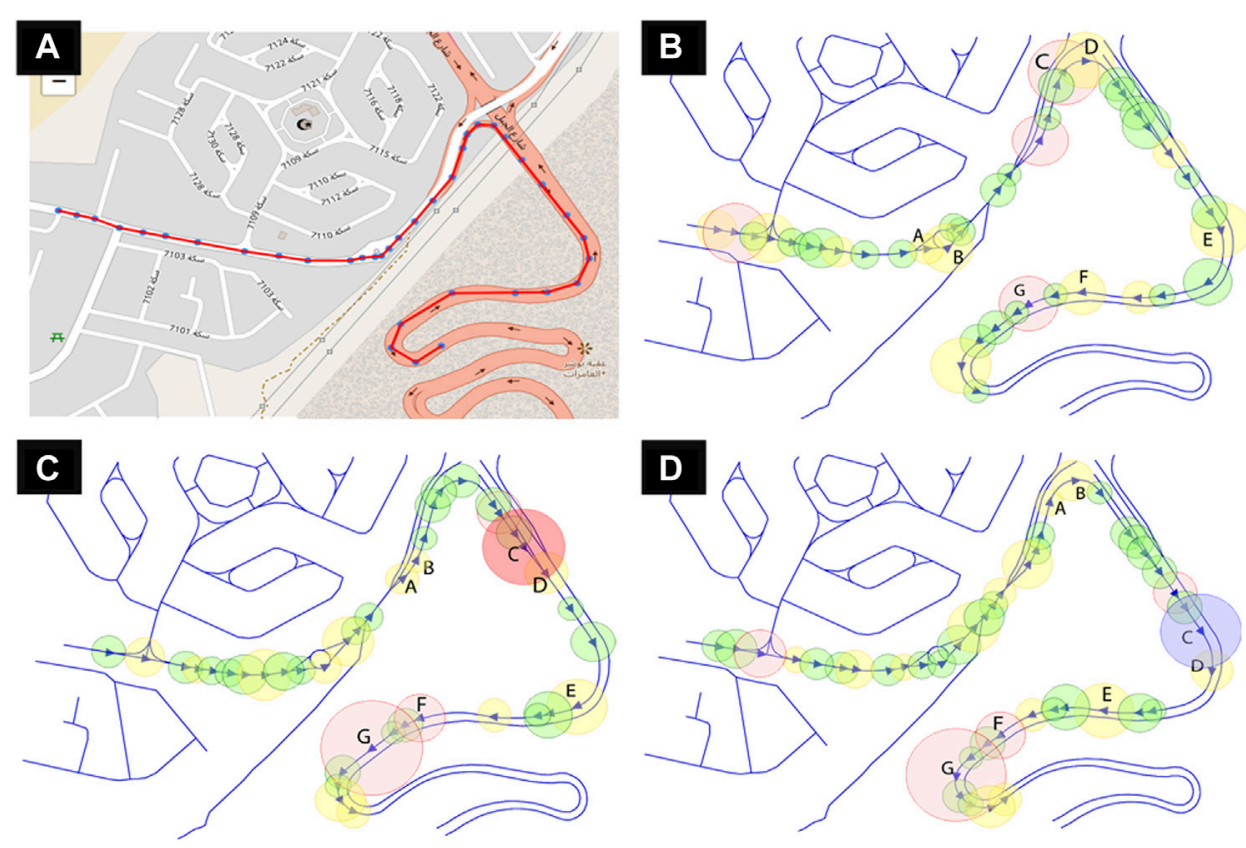

FIGURE 5 | (A) Road section for our field tests; (B-D) Snapshots of three consecutive driving situations simulated with software agents.
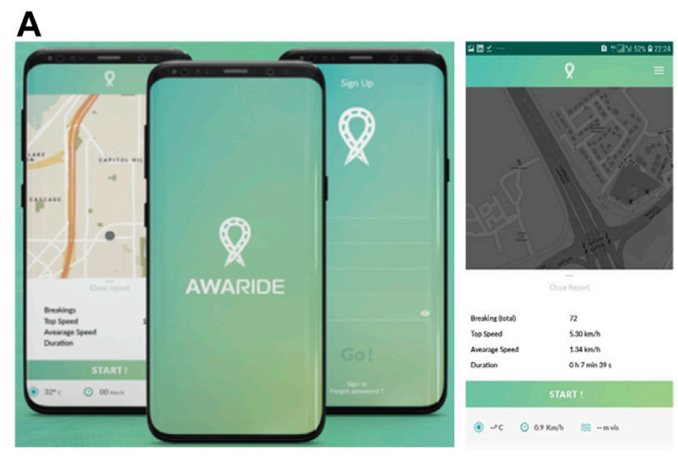

B

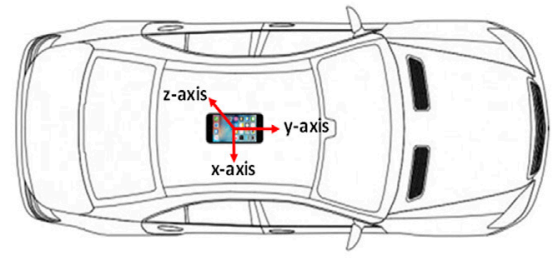

FIGURE 6 | (A) The AWARIDE mobile app; (B) Orientation of the smartphone with respect to the vehicle.

the wider the notification range). Based on our simulations, our agents are capable of assessing their respective drivers' behaviors, finding out the driving actions to avoid, and identifying the appropriate driving styles. They are also capable of exchanging appropriate data with agents representing CVs accordingly. In addition, our agents are capable of recognizing and implementing personalized policies related to speed limits.

Our field tests were carried out with a Toyota Camry vehicle (model 2009) with a manual transmission gear. We did 20 trips with the test car on the selected path depicted on Figure 5A. We developed a dedicated mobile app called AWARIDE (see Figure 6A) and installed it on a smartphone Galaxy J7 Pro. As smartphone sensors are not adjusted by default to collect data without noise, several approaches [e.g., (Vlahogianni and Barmpounakis 2017; Bergasa, Almazán, and Arroyo 2014; Fazeen et al., 2012)] have been proposed to calibrate them before collecting any readings about driving behaviors. These approaches have focused on identifying the appropriate positioning of the smartphone inside the vehicle. Some of them have proposed to match the orientation of the device with the one of the vehicle (e.g., Fazeen et al., 2012). Other approaches [e.g. (Vlahogianni and Barmpounakis 2017)] have proposed a dynamically updated reorientation algorithm which automatically corrects the sensors' signals and removes the uncertainties resulting from the random positioning of smartphones inside the vehicles. Within the context of this research work, we decided to fix the smartphone on the roof of the vehicle and match their respective orientations as depicted on Figure 6B. Our decision is based on recommendations from several studies [e.g., (Fazeen et al., 2012)]. In this orientation, the $y$-axis is pointing toward the front of the vehicle to measure the accelerations and the braking, the $x$-axis is pointing to the right of 

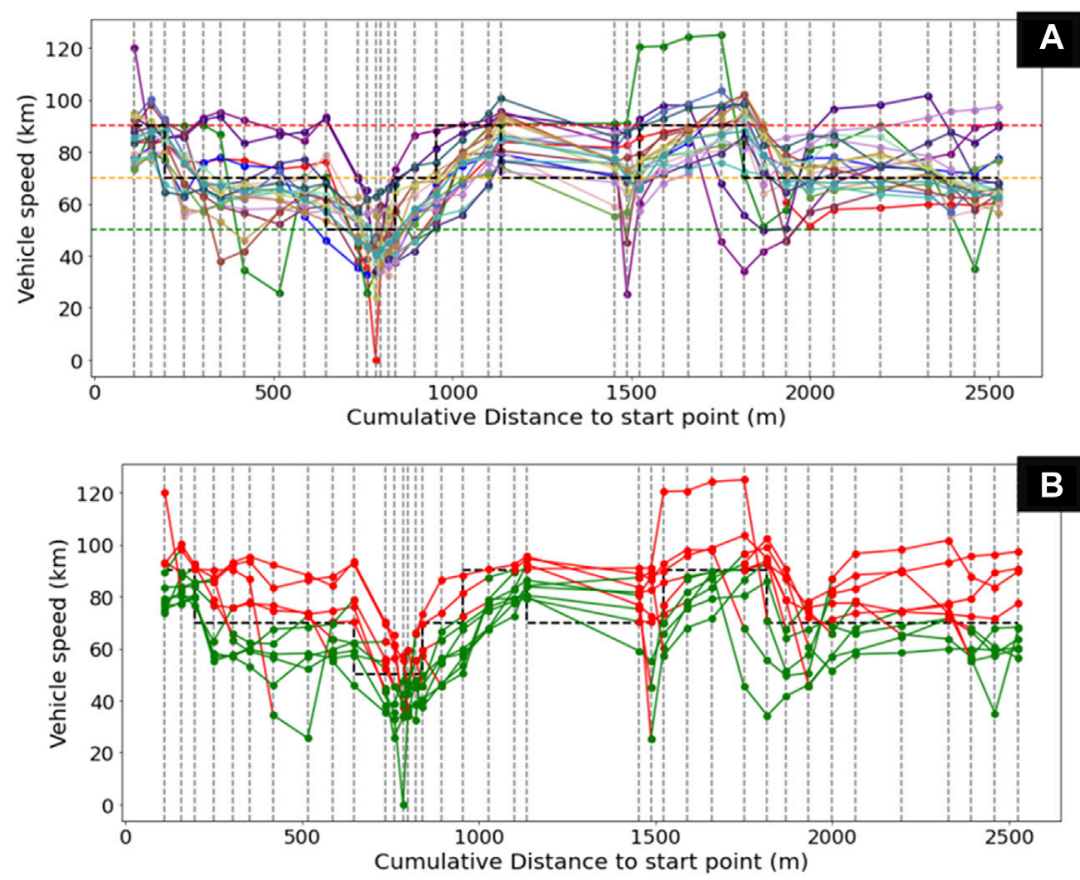

FIGURE 7 | Illustration of the speed variations of some trips with to speed limit.

the vehicle to report on the turning and lane changes, and the $z$-axis is pointing up to measure the vibrations as well as the road anomalies.

In order to calculate the longitudinal acceleration accLong of the vehicle from the readings obtained through the AWARIDE app, we used the following expression:

$$
\text { accLong }=\frac{\Delta \mathrm{v}}{\Delta \mathrm{t}}
$$

Where $\Delta \mathrm{v}$ and $\Delta \mathrm{t}$ denote the variation of speed and time respectively. The value of $\Delta t$ is $1 \mathrm{~s}$ since the GPS has a sampling frequency of $1 \mathrm{~Hz}$.

Likewise, the lateral acceleration accLat is calculated as follows:

$$
\text { accLat }=\frac{v^{2}}{\mathrm{R}}
$$

Where $\mathrm{R}$ is the radius of the turn $(\mathrm{m})$ and $\mathrm{v}$ is the current speed $(\mathrm{m} / \mathrm{s})$. The value of $\mathrm{R}$ can be estimated using the following expression (Abdulrahim 2006):

$$
R=\frac{(180 . v)}{\pi \cdot \Delta \text { Heading }}
$$

Where $\pi . \Delta$ Heading refers to the change of the heading in the temporal unit. The heading (expressed in degree) denotes the current direction of the vehicle with respect to the North direction (the rotation toward the East is positive).

We report on Figure 7.a some trips to showcase the variation of driving speeds with respect to the limit of speeds along the selected test road section. On Figure $\mathbf{7 B}$, we highlight with red color the driving records which are above the speed limit and with green color the driving records which comply with the regulatory framework.

A zoom in on speed variations is illustrated on Figure 8, where the $X$ and $Y$ axis represent the data collection points and the speeds in these points, respectively. The $Z$ axis represents the variations with respect to the speed limit (i.e., actual speed - speed limit) in Figures 8A,C. In Figures 8B,D, the $Z$ axis reports on the penalties calculated in every data collection point. The visualization of these details are important to the driver for BSR (i.e., Behavioral Self-Regulation) reactions. On Figure $\mathbf{8 A}$, the actual speed is excessively higher than the limit speed in some areas. Nevertheless, the corresponding penalties are not considered to be high (Figure 8B). This could be explained by the fact that data are reported for the early trips. On Figure $\mathbf{8 C}$, the variation of the actual speed with respect to the speed limit is different from Figure 8A, which is normal as trips are independent. However, the penalties (Figure 8D) are higher than those in Figure 8B. This can be explained by the fact that since the penalties are cumulative, the previous behavior is affecting the current one. Once the penalties reach a predefined threshold, the test vehicle issues a notification using the mobile app AWARIDE. As for the current prototype, we are capable of assessing the driving behaviors, identifying personalized policies with respect to speed limit, and generating warning messages. However, we did not test the exchange of data between CVs as well as their impact on driving behaviors.

\section{CONCLUSION}

The advent of Connected Vehicles (CVs) is creating new opportunities toward safer and more effective commutes. 

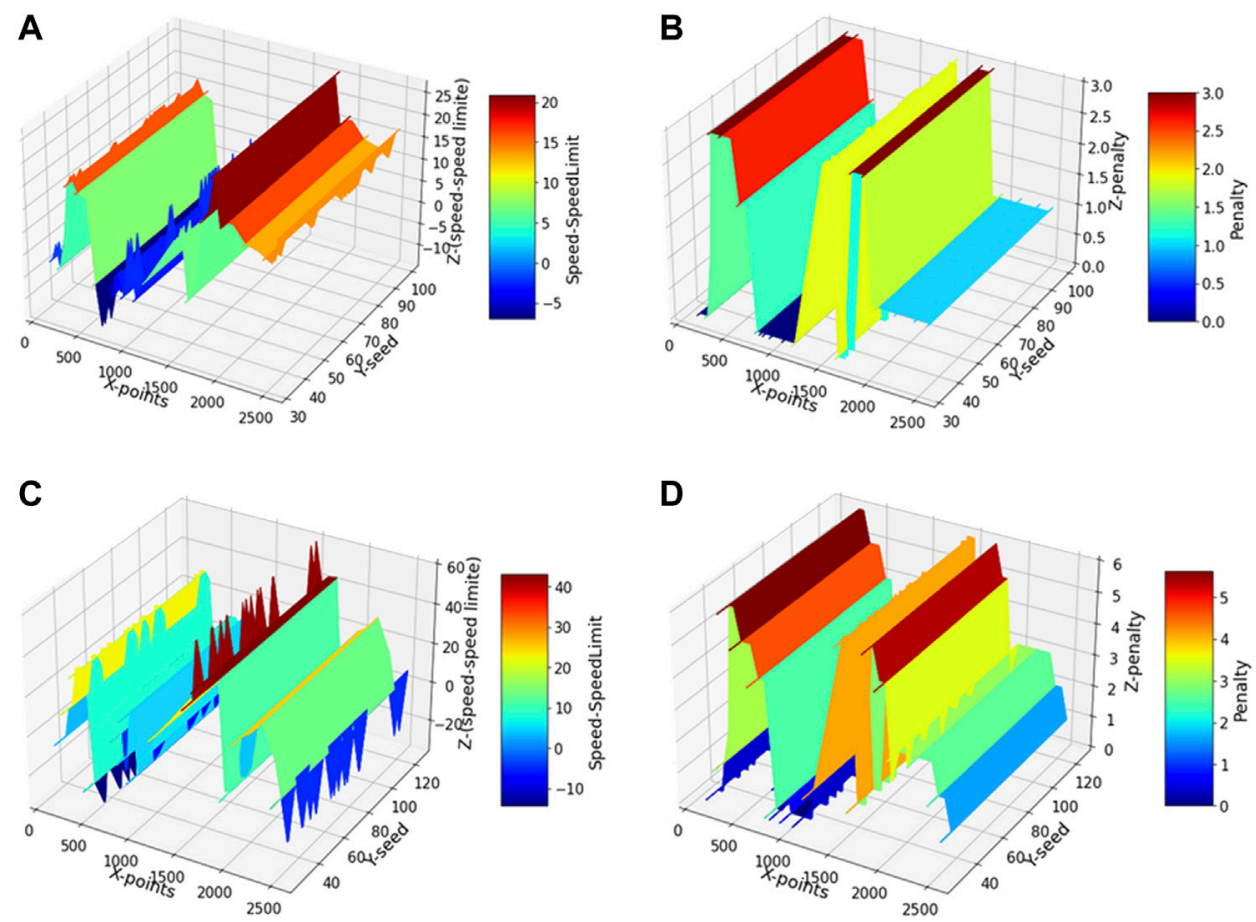

FIGURE 8 | Visualization of driving performance with respect to speed limits and penalties.

More precisely, road traffic crashes are expected to decrease, the use of the available road infrastructures is being optimized, and the environmental impact of road traffic is predicted to diminish. Several studies have highlighted that these benefits are going to be obtained via the expected positive influence of CVs on driving behaviors. We focused in this paper on the investigation of this influence from a policy perspective. More precisely, we highlighted the need for a road traffic framework within which personalized policies will be identified and applied to specific drivers based on their driving performance. Indeed, as vehicles do not have the same capabilities and drivers do not have the same attributes, some road policies should be adapted accordingly while remaining under the umbrella of the national regulatory framework. To meet our goals, we proposed a well-defined set of driving states that are identified based on driving behaviors and performance. The transition between these states is then managed by an agent-based solution which is driven by a slightly modified BDI architecture where undesirable and/or prohibited actions represent the main focus. The solution is responsible of identifying the relevant data to be shared among CVs to ultimately enhance mobility and increase road traffic safety. It is also promoting Behavioral Self-Regulation actions to increase awareness and impact driving behaviors. In order to showcase the performance of our solution, we run software simulations as well as field tests.

The initial results obtained are promising. However, some limitations still need to be addressed. More precisely, our future works will focus on further investigating the notion of personalized policies as well as their impact on driving behaviors. We are also going to transform the software agents representing road sections into intelligent and proactive entities and assess any impact their behaviors may have on the identification of personalized driving policies. Furthermore, we are going to address the current limitation of our work concerning the impact of the proposed methodology within the context of connected vehicles. We will ultimately aim to explore if the connected-vehicle environment may result into additional legal driving states. In order to confirm the findings of our future works, we will carry extensive field tests based an extended new version of our mobile app AWARIDE that will be deployed on several vehicles.

\section{DATA AVAILABILITY STATEMENT}

The raw data supporting the conclusions of this article will be made available by the authors, without undue reservation.

\section{AUTHOR CONTRIBUTIONS}

All authors listed have made a substantial, direct, and intellectual contribution to the work and approved it for publication.

\section{FUNDING}

This work was partially supported and funded by the Research Cluster \#R17075 of the Zayed University, United Arab Emirates. 


\section{REFERENCES}

Abdulrahim, M. (2006). On the Dynamics of Automobile Drifting. Society of Automotive Engineers. doi:10.4271/2006-01-1019

Abuali, N., and Abou-Zeid, H. (2016). Driver Behavior Modeling: Developments and Future Directions. Int. J. Vehicular Technology 2016, 1-12. doi:10.1155/ 2016/69527912016

Ali, Y., Sharma, A., Haque, M. M., Zheng, Z., and Saifuzzaman, M. (2020). The Impact of the Connected Environment on Driving Behavior and Safety: A Driving Simulator Study. Accid. Anal. Prev. 144 (May), 105643. doi:10.1016/j. aap.2020.105643

Alkinani, M. H., Khan, W. Z., and Arshad, Q. (2020). Detecting Human Driver Inattentive and Aggressive Driving Behavior Using Deep Learning: Recent Advances, Requirements and Open Challenges. IEEE Access 8, 105008-105030. doi:10.1109/ACCESS.2020.2999829

Annual Accident Report (2017). Annual Accident Report 2017. Available at: https:// ec.europa.eu/transport/road_safety/sites/roadsafety/files/pdf/statistics/dacota/ asr2017.pdf (Accessed March 10, 2021).

Baker, R. T., and Wagner., J. (2013). "Policy Pathways to Vehicle Automation: Industry Perspectives on the Role of Public Policy in Autonomous Vehicle Development," in 2013 International Conference on Connected Vehicles and Expo, ICCVE 2013 - Proceedings, Las Vegas, NV, December 2-6, 2013, 431-436. doi:10.1109/ICCVE.2013.6799831

Bergasa, Luis. Miguel., Almazán, Javier., and Arroyo, Roberto. (2014). DriveSafe: An App for Alerting Inattentive Drivers and Scoring Driving Behaviors SIAMO (Aided Movility with an Integral System) View Project Smart Elderly Car View Project. 2014 IEEE Intell. Vehicles Symp. Proc. (Iv), 240-245.

Birrell, S. A., Fowkes, M., and Jennings, P. A. (2014). Effect of Using an In-Vehicle Smart Driving Aid on Real-World Driver Performance. IEEE Trans. Intell. Transport. Syst. 15 (4), 1801-1810. doi:10.1109/TITS.2014.2328357

Castro, Daniel. (2011). Benefits and Limitations of Industry Self-Regulation for Online Behavioral Advertising. Washington, DC: The Information Technology and Innovation Foundation.

Chan, T. K., Chin, C. S., Chen, H., and Zhong, X. (2020). A Comprehensive Review of Driver Behavior Analysis Utilizing Smartphones. IEEE Trans. Intell. Transport. Syst. 21 (10), 4444-4475. doi:10.1109/TITS.2019.2940481

Chang, X., Li, H., Rong, J., Huang, Z., Chen, X., and Zhang, Y. (2019). Effects of On-Board Unit on Driving Behavior in Connected Vehicle Traffic Flow. J. Adv. Transportation 2019, 1-12. doi:10.1155/2019/8591623

Choi, H. J., Lee, S., No, S.-R., and Kim, E. I. (2016). Effects of Compassion on Employees' Self-Regulation. Soc. Behav. Pers 44 (7), 1173-1190. doi:10.2224/ sbp.2016.44.7.1173

D’Angelo, G., Castiglione, A., and Palmieri, F. (2020). A Cluster-Based Multidimensional Approach for Detecting Attacks on Connected Vehicles. IEEE Internet Things J. 4662 (c), 1. doi:10.1109/jiot.2020.3032935

Djahel, S., Jabeur, N., Nait-Abdesselam, F., and Wolstencroft, T. (2020). A WAVE Based and Collaboration Driven Framework for Reduced Traffic Congestion in Smart Cities. IEEE Intell. Transport. Syst. Mag. 2020, 2-12. doi:10.1109/MITS. 2019.2953487

Doğan, Ebru. Burcu., Rothengatter, Talib., Steg, Linda., and Delhomme, Patricia. (2011). "Self-Regulation and Driving Behavior," in In Traffic Psychology. Editor Dwight. Hennessy (Hauppauge, NY, USA: Nova Science Publishers, Inc), 129-143.

Dong, S., Chen, H., Yang, Z., Liu, Q., and Wang, P. (20192019). A Hierarchical Strategy for Velocity Optimization of Connected Vehicles with the Existence of Multiple Traffic Lights. CCC (1), 6680-6685. doi:10.23919/ChiCC.2019. 8865236

Ezell, S., and Atkinson, R. D. (2015). From Concrete to Chips : Bringing the Surface Transportation Reauthorization Act into the Digital Age. http://www2.itif.org/ 2015-concrete-to-chips.pdf.

Fazeen, M., Gozick, B., Dantu, R., Bhukhiya, M., and González, M. C. (2012). Safe Driving Using Mobile Phones. IEEE Trans. Intell. Transport. Syst. 13 (3), 1462-1468. doi:10.1109/tits.2012.2187640

Feraud, M., and Galland, S. (2017). First Comparison of SARL to Other AgentProgramming Languages and Frameworks. Proced. Computer Sci. 109, 1080-1085. doi:10.1016/j.procs.2017.05.389
Gao, Y., and Chen, J. (2019). The Risk Reduction and Sustainable Development of Shared Transportation: The Chinese Online Car-Hailing Policy Evaluation in the Digitalization Era. Sustainability 11 (9), 2596. doi:10.3390/su11092596

Goli, S. A., Far, B. H., and Fapojuwo, A. O. (20182018). Vehicle Trajectory Prediction with Gaussian Process Regression in Connected Vehicle Environment\$\star\$. Proceedings (Iv), 550-555. doi:10.1109/IVS.2018.8500614

Gong, S., and Du, L. (2018). Cooperative Platoon Control for a Mixed Traffic Flow Including Human Drive Vehicles and Connected and Autonomous Vehicles. Transportation Res. B: Methodological 116, 25-61. doi:10.1016/j.trb.2018. 07.005

Hussain, R., and Zeadally, S. (2019). Autonomous Cars: Research Results, Issues, and Future Challenges. IEEE Commun. Surv. Tutorials 21 (2), 1275-1313. doi:10.1109/COMST.2018.2869360

Ilgin Guler, S., Menendez, M., and Meier, L. (2014). Using Connected Vehicle Technology to Improve the Efficiency of Intersections. Transportation Res. C: Emerging Tech. 46, 121-131. doi:10.1016/j.trc.2014.05.008

Jabbar, Rateb., Shinoy, Mohammed., Kharbeche, Mohamed., Al-Khalifa, Khalifa., Krichen, Moez., and Barkaoui, Kamel. (2020). Driver Drowsiness Detection Model Using Convolutional Neural Networks Techniques for Android Application. ArXiv 237-242.

Jadaan, K., Zeater, S., and Abukhalil, Y. (2017). Connected Vehicles: An Innovative Transport Technology. Proced. Eng. 187, 641-648. doi:10.1016/j.proeng.2017. 04.425

Kesting, A., Treiber, M., and Helbing, D. (2010). Enhanced Intelligent Driver Model to Access the Impact of Driving Strategies on Traffic Capacity. Phil. Trans. R. Soc. A. 368, 4585-4605. doi:10.1098/rsta.2010.0084

Lee, Dave. (2021). Autonomous Vehicle Implementation Predictions: Implications for Transport Planning. Transportation Res. Board Annu. Meet. 42.

Lee, K., Yoon, H., Song, J., and Park, K. (2018). Convolutional Neural NetworkBased Classification of Driver's Emotion during Aggressive and Smooth Driving Using Multi-Modal Camera Sensors. Sensors 18 (4). doi:10.3390/ s18040957

Mbarek, B., Jabeur, N., Pitner, T., and Yasar, A.-U. -H. (2020). Empowering Communications in Vehicular Networks with an Intelligent Blockchain-Based Solution. Sustainability 12 (19). doi:10.3390/SU12197917

Mcquinn, Alan., and Castro, Daniel. (2018). “A Policymaker's Guide to Connected Cars. doi:10.26504/qec2018aut

Nagamachi, M. (2003). Kansei Engineering. Occup. Ergon. Des. Management Work Syst. 15, 25-1-25-14. doi:10.1201/978020301045710.1201/9780203010457.sec4

Norling, Emma. (2004). "Folk Psychology for Human Modelling: Extending the BDI Paradigm," in Proceedings of the Third International Joint Conference on Autonomous Agents and Multiagent Systems, AAMAS 2004, 202-209.

Pariota, L., Bifulco, G. N., Markkula, G., and Romano, R. (2017). Validation of Driving Behaviour as a Step towards the Investigation of Connected and Automated Vehicles by Means of Driving Simulators. 5th IEEE Int. Conf. Models Tech. Intell. Transportation Syst. MT-ITS 2017 - Proc., 274-279. doi:10. 1109/MTITS.2017.8005679

Park, J., Abdel-Aty, M., Wu, Y., and Mattei, I. (2019). Enhancing In-Vehicle Driving Assistance Information under Connected Vehicle Environment. IEEE Trans. Intell. Transport. Syst. 20 (9), 3558-3567. doi:10.1109/TITS.2018. 2878736

Qi, X., Sun, T., Ma, W., Wang, L., and Yu, C. (2020). Evaluation of a Variable Speed Limit System Using a Hierarchical Framework under a Connected Vehicles Environment: A Case Study of a Field Test in Seattle. IET Intell. Transport Syst. 14 (9), 1125-1131. doi:10.1049/iet-its.2019.0858

Sanguinetti, A., Queen, E., Yee, C., and Akanesuvan, K. (2020). Average Impact and Important Features of Onboard Eco-Driving Feedback: A Meta-Analysis. Transportation Res. F: Traffic Psychol. Behav. 70, 1-14. doi:10.1016/j.trf. 2020.02.010

Sharma, A., Ali, Y., Saifuzzaman, M., Zheng, Z., and Haque, M. M. (2018). Human Factors in Modelling Mixed Traffic of Traditional, Connected, and Automated Vehicles. Adv. Intell. Syst. Comput. 591, 262-273. doi:10.1007/978-3-31960591-3_24

Talebpour, A., and Mahmassani, H. S. (2016). Influence of Connected and Autonomous Vehicles on Traffic Flow Stability and Throughput. Transportation Res. Part C: Emerging Tech. 71, 143-163. doi:10.1016/j.trc. 2016.07.007 
Tampère, C. M. J., Hoogendoorn, S. P., and Van Arem, B. (2009). Continuous Traffic Flow Modeling of Driver Support Systems in Multiclass Traffic with Intervehicle Communication and Drivers in the Loop. IEEE Trans. Intell. Transport. Syst. 10 (4), 649-657. doi:10.1109/TITS.2009.2026442

Tanveer, H., Mubasher, M. M., and Jaffry, S. W. (2020). Integrating Human Panic Factor in Intelligent Driver Model. 3rd Int. Conf. Advancements Comput. Sci. ICACS 2020, 1-6. doi:10.1109/ICACS47775.2020.9055947

Toledo, T. (2007). Driving Behaviour: Models and Challenges. Transport Rev. 27 (1), 65-84. doi:10.1080/01441640600823940

Vaezipour, A., Rakotonirainy, A., Haworth, N., and Delhomme, P. (2017). Enhancing Eco-Safe Driving Behaviour through the Use of In-Vehicle Human-Machine Interface: A Qualitative Study. Transportation Res. A: Pol. Pract. 100, 247-263. doi:10.1016/j.tra.2017.04.030

Vlahogianni, E. I., and Barmpounakis, E. N. (2017). Driving Analytics Using Smartphones: Algorithms, Comparisons and Challenges. Transportation Res. Part C: Emerging Tech. 79, 196-206. doi:10.1016/j.trc.2017.03.014

Vohs, K. D., Baumeister, R. F., and Ciarocco, N. J. (2005). Self-Regulation and SelfPresentation: Regulatory Resource Depletion Impairs Impression Management and Effortful Self-Presentation Depletes Regulatory Resources. J. Personal. Soc. Psychol. 88 (4), 632-657. doi:10.1037/0022-3514.88.4.632
Yu, Z., Hu, J., Min, G., Zhao, Z., Miao, W., and Hossain, M. S. (2020). MobilityAware Proactive Edge Caching for Connected Vehicles Using Federated Learning. IEEE Trans. Intell. Transport. Syst., 1-11. doi:10.1109/tits.2020. 3017474

Zhu, X., Hu, X., and Chiu, Y.-C. (2013). Design of Driving Behavior Pattern Measurements Using Smartphone Global Positioning System Data. Int. J. Transportation Sci. Technology 2 (4), 269-288. doi:10.1260/2046-0430.2. 4.269

Conflict of Interest: The authors declare that the research was conducted in the absence of any commercial or financial relationships that could be construed as a potential conflict of interest.

Copyright (c) 2021 Outay, Jabeur, Haddad, Bouyahia and Gharrad. This is an openaccess article distributed under the terms of the Creative Commons Attribution License (CC BY). The use, distribution or reproduction in other forums is permitted, provided the original author(s) and the copyright owner(s) are credited and that the original publication in this journal is cited, in accordance with accepted academic practice. No use, distribution or reproduction is permitted which does not comply with these terms. 